\title{
High Performance self-injection locked 524 nm green laser diode for high bitrate visible light communications
}

\author{
M. Hosne M. Shamim¹, M. A. Shemis ${ }^{1}$, Chao Shen², Hassan M. Oubei², Tien Khee Ng$^{2}$, Boon S. Ooi ${ }^{2}$, \\ M. Z. M. Khan ${ }^{*}$ \\ ${ }^{\prime}$ Optoelectronic Research Laboratory, Department of Electrical Engineering, King Fahd University of Petroleum \& Minerals (KFUPM), \\ Dhahran 31261, Saudi Arabia \\ ${ }^{2}$ Computer, Electrical and Mathematical Sciences and Engineering (CEMSE) division, King Abdullah University of Science \& Technology \\ (KAUST), Thuwal 23955-6900, Saudi Arabia \\ "zahedmk@kfupm.edu.sa
}

\begin{abstract}
First demonstration of self-injection locking on $524 \mathrm{~nm}$ visible laser diode is presented. Enhancement by $\sim 440 \mathrm{MHz}(\sim 30 \%)$ in modulation bandwidth, $\sim 7$ times reduction in lasing linewidth, and $\sim 10 \mathrm{~dB}$ improvement in SMSR is achieved.
\end{abstract}

\section{Introduction}

Emerging of new smart technologies make our life easier but the price for that comfort is a huge data traffic which our present infrastructure is not capable of handling. Although, we have high speed optical fiber internet backbone under the ocean, because of the limitations of radio frequency (RF) technology, the low speed at the consumer end is posing a challenge. Recently, visible light optical wireless communication (VLC) has been identified as a promising solution enabling simultaneous high speed communication and lighting. In this regard, semiconductor laser diodes (LDs) are proving to be more suitable than LED as the transmitter in a VLC communication link, thanks to the improved dynamic characteristics.

The modulation bandwidth and spectral linewidth are the two key parameters that fundamentally dictates the modulation capability of LDs and hence the capacity of an optical communication system. For instance, Bragg gratings, distributive feedback, optical injection locking, etc., have been considered in near infra-red (NIR) based light sources to achieve almost single wavelength operation with transmission data rates in 10 's of Gb/s using direct modulation scheme. In particular, optical injection locking is found to be an attractive assisting solution where the frequency of the external light is coincident with one of the slave laser resonator modes, thereby locking of that particular mode, which leads to significant reduction of the linewidth [1] and improvement of the side mode suppression ratio (SMSR). Two variations of injection locking have been investigated; external injection locking (EIL) and self-injection locking (SIL). While the former utilizes an external seeding master light source, the latter is based on self-feeding part of the laser power back into the device for locking purpose that significantly reduces the system cost and provides robustness against environmental conditions (self-aligned), the temperature in particular.

Although injection locking has been comprehensively studied and the underlying physics is well understood in the NIR LDs, they are living in their infancy in the visible region with a handful of reports in the literature. For instance, Lu et al. [2] demonstrated 2.4 times improvement in modulation bandwidth using EIL scheme on $680 \mathrm{~nm}$ red vertical cavity surface emitting laser (VCSEL) and successfully transmitted $20 \mathrm{~Gb} / \mathrm{s}$ 16-quadrature amplitude modulated (16-QAM) orthogonal frequency division multiplexed (OFDM) signal via direct modulation [2]. The work was further extended to include two stage EIL to demonstrate $56 \mathrm{~Gb} / \mathrm{s} \mathrm{LiFi}$ transmission using pulse amplitude modulation (PAM-4) scheme [3]. Later, incorporation of both optical and electronic injection was investigated with a demonstration of $\sim 2.9$ times improvement in the bandwidth [4] and $16 \mathrm{~Gb} / \mathrm{s}$ underwater optical wireless communication at $488 \mathrm{~nm}$ [5]. In general, all these reports employed EIL scheme aiming communication performance improvement, and hence a comprehensive locking characteristics is lacking. Besides, self-injection locking on visible semiconductor lasers has not been reported in the literature, to our knowledge.

In this work, we comprehensively investigated self-injection locking technique on InGaN/GaN $524 \mathrm{~nm}$ commercially available green LD for the first time and demonstrated an improvement of $\sim 1.3$ times in the modulation bandwidth besides significantly reducing the lasing linewidth. We strongly believe that our results would open a new research paradigm for achieving low cost and robust VLC system, without sacrificing the communication performance.

\section{Experimental Setup}

Fig. 1 shows the self injection locking experimental setup. A commercial single mode TO can green laser diode (Thorlab L520P50) and a $7 \mathrm{GHz}$ silicon PIN Ultrafast Photo Detector (UPD) from Alphalas are employed as a transmitter and receiver, respectively. The threshold current $\left(\mathrm{I}_{\mathrm{th}}\right)$ of the LD is measured to be $34 \mathrm{~mA}$ and found to 
reduces to $33.7 \mathrm{~mA}$ under self-optical feedback mode. This is attributed to the enhancement of the photon density in the active region of the LD after the optical feedback, which has been observed in the literature [1], [2] and considered as one of the signatures of injection locking. The transmitted beam is collimated by an anti-reflection coated aspheric lens (Lens 1, Thorlabs A375TM-B) of focal length $7.5 \mathrm{~mm}$ with a reflectance of $0.25-0.5$, and passed through a $92: 8 \%$ pellicle beam splitter (BS, Thorlabs BP108) to enable simultaneous self-optical feedback and performance characterization. Another identical 92:8\% BS has been used to observe the lasing spectrum on 0.05 $\mathrm{nm}$ resolution Optical Spectrum Analyzer (OSA, Yokogawa AQ6373B) after focusing through a plano-convex lens (Lens 3, Thorlabs LA4148) of focal length $50 \mathrm{~mm}$. The rest of the $92 \%$ of the beam goes through a bi-convex lens (Lens 2, Thorlabs LB1471) of the same focal length to the UPD with responsivity $0.235 \mathrm{~A} / \mathrm{W}$ at $524 \mathrm{~nm}$. Because of the low sensitivity of the detector, a $26 \mathrm{~dB}$ linear amplifier (Tektronix PSPL5865) is employed to enhance the modulated signal before analyzing on $67 \mathrm{GHz}$ Network Analyzer (Agilent E8361C). A silver coated mirror whose reflectivity is $97.5 \%$ in the green emitting region, has been used to facilitate the self-optical feedback to the system, thus forming a $\sim 56 \mathrm{~cm}$ external cavity between the LD facet and the mirror. For the ease of alignment and to block the scattered light, three post-mountable iris diaphragms are employed in the system. All the measurements are investigated at a fixed $20^{\circ} \mathrm{C}$ heat-sink temperature.
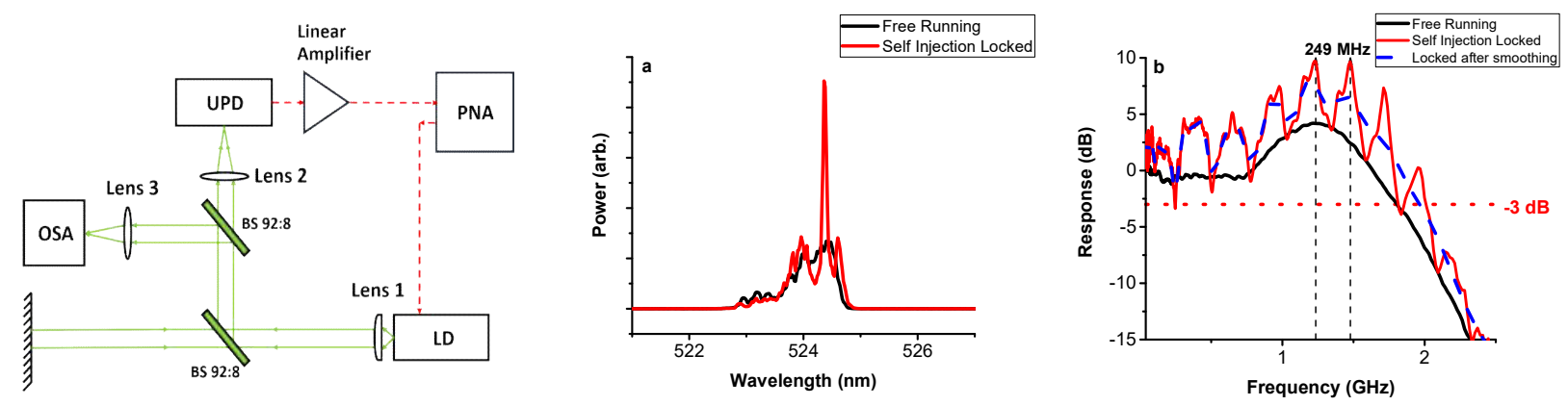

Fig. 1. Experimental setup of self-injection locking system forming a $\sim 56 \mathrm{~cm}$ external cavity. The green lines correspond to the optical path and the red-dashed lines correspond to the electrical path.

Fig. 2. (a) The free running and self-injection locked lasing spectrum at an injection of $80 \mathrm{~mA}$, and (b) the corresponding frequency responses, of $524 \mathrm{~nm}$ green TO can edge-emitting LD. The dashed black vertical lines in (b) correspond to frequency separation of the external cavity, and the horizontal red dashed line is the $-3 \mathrm{~dB}$ line.

\section{Results and Discussion:}

The lasing spectrum and normalized frequency response of the LD at $80 \mathrm{~mA}\left(2.35 \mathrm{I}_{\mathrm{th}}\right)$ are presented in Fig. 2. A clear locking signature is evident from Fig. 2(a) when compared to the free running case, wherein one particular Fabry-Perot (FP) mode is found to dominate. The injection ratio, in this case, is estimated to be $\sim-4 \mathrm{~dB}$. Modulations in the frequency response curve, forming peaks and valleys, is observed in the locked case, while a typical curve is observed for the free running case. This is attributed to the external cavity formation under SIL in the system. In fact, the difference between any two consecutive peaks is $\sim 249 \mathrm{MHz}$ which is in excellent agreement to the calculated frequency spacing of $\sim 268 \mathrm{MHz} \mathrm{MHz}$ for the $\sim 56 \mathrm{~cm}$ external cavity, i.e. $\Delta v=c / 2 n L$ where $c$ is the speed of light, $n=1$, and $L=\sim 56 \mathrm{~cm}$ are the refractive index and length of the external cavity, respectively. Moreover, a considerable improvement in the system response by $3.5-6 \mathrm{~dB}$ is also observed, thus potentially facilitating higher direct modulation data transmission. The fluctuations in the frequency response at lower frequencies are consistent with the literature and has been termed as 'Low frequency Fluctuations (LFF) [6]. Besides, a stable locking region with injection ratio $>-10 \mathrm{~dB}$ [7] has been observed to be possible under LFF, which is exactly what has been observed in our case.

In Fig. 3 (a), we plot the improvement in the modulation bandwidth in the locked case compared to the free running case at different current injections while keeping the injection ratio fixed $\sim-4 \mathrm{~dB}$. It can be seen that a maximum improvement of $\sim 440 \mathrm{MHz}$, which corresponds to $\sim 1.3$ times of the free running value, is achieved at an injection current of $120 \mathrm{~mA}\left(3.53 \mathrm{I}_{\mathrm{th}}\right)$. The change in the linewidth and SMSR of the LD before and after locking are illustrated in Fig. 3 (b) and (c), respectively. In general, the linewidth is found to increase with higher injection current in the free running case and followed by the locked case, which is attributed to the increase in the number of longitudinal modes attaining threshold condition. For instance, an improvement in the linewidth of $\sim 2$ times $(\sim 0.8$ $\mathrm{nm}$ to $0.4 \mathrm{~nm})$ is observed at $120 \mathrm{~mA}$ while it is $\sim 7$ times $(\sim 0.7 \mathrm{~nm}$ to $0.1 \mathrm{~nm})$ at $80 \mathrm{~mA}$. In addition, the SMSR plotted in Fig. 3(c) exhibits the similar trend. A maximum of $\sim 10 \mathrm{~dB}$ improvement at $40 \mathrm{~mA}$ with the value 
decreasing to $\sim 5 \mathrm{~dB}$ at $80 \mathrm{~mA}$ and eventually to $\sim 1 \mathrm{~dB}$ at $120 \mathrm{~mA}$. We postulate that higher quantum efficiency at lower injection might be the reason behind this observation. More experiments are underway to shed physical insight to these observations and further improve the dynamic characteristics of the laser for high speed VLC.
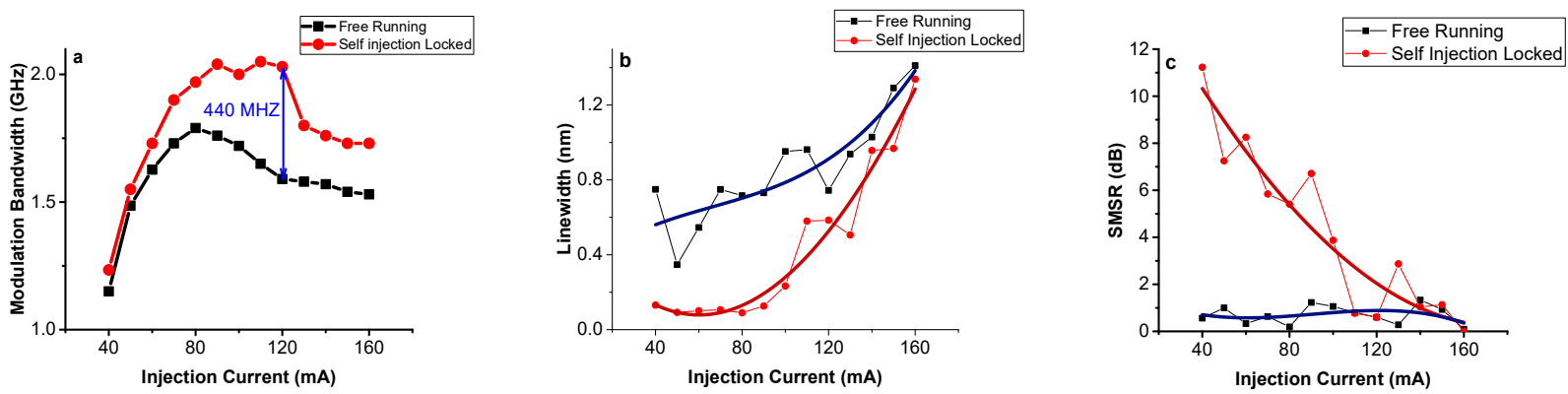

Fig. 3. Comparison of (a) modulation bandwidth, (b) spectral linewidth, and (c) SMSR between the free running and self injection locked $524 \mathrm{~nm}$ TO can laser diode at different injection currents. A considerable improvement of $\sim 1.3$ times in the bandwidth is achieved in the locking case.

\section{Conclusion:}

Self-injection locking technique is demonstrated on a commercial $524 \mathrm{~nm}$ green laser diode, for the first time, employing a simple architecture. The signature of locking is affirmed by exhibiting improvement in the modulation bandwidth by $\sim 30 \%$, reduction in the spectral linewidth by $\sim 7$ times and enhancement in SMSR by $\sim 10 \mathrm{~dB}$. Further characterization and communication experiments are underway. We strongly believe that self-seeding could potentially provide a new way to improve visible LDs performance characteristics for eventual achievement of high data rate visible light communication in the form of $\mathrm{LiFi}$, underwater optical wireless communication, etc.

\section{Acknowledgment}

MHMS, MAS, and MZMK gratefully acknowledges the support from King Fahd University of Petroleum and Minerals (KFUPM) through the research grant KAUST004. The authors also acknowledge funding support from King Abdulaziz City for Science and Technology (KACST) Technology Innovation Center (TIC) for Solid State Lighting (KACST TIC R2-FP- 008). BSO, TKN, HMO, and CS acknowledge KAUST baseline funding (BAS/1/1614-01-01).

\section{References}

[1] W. Liang et al., "Ultralow noise miniature external cavity semiconductor laser," Nat. Commun., vol. 6, p. 7371, 2015.

[2] C.-L. Ying, H. Lu, C. Li, C. Cheng, P. Peng, and W. Ho, "20-Gbps optical LiFi transport system.," Opt. Lett., vol. 40, no. 14, pp. 32769, 2015.

[3] X.Y. Lin et al., "A 56-Gbps PAM4 LiFi Transmission System Based on VCSEL with Two-Stage Injection-Locked Technique,” in Optical Fiber Communication Conference and Exhibition (OFC), 2017, pp.1-3.

[4] H. Lu et al., "45 Gb/s PAM4 transmission based on VCSEL with light injection and optoelectronic feedback techniques," Opt. Lett., vol. 41 , no. 21 , p. 5023-6, 2016

[5] C. Li et al., " $16 \mathrm{~Gb} / \mathrm{s}$ PAM4 UWOC system based on 488-nm LD with light injection and optoelectronic feedback techniques," Opt. Express, vol. 25, no. 10, p. 11598, 2017.

[6] J. Mork et al., "Bistability and Low-Frequency Fluctuations in Semiconductor Lasers with Optical Feedback : A Theoretical Analysis," IEEE Journal of Quantum Electronics, vol. 24, no. 2, pp. 123-133, 1988

[7] T. Heil, I. Fischer, and W. Elsa, "Coexistence of low-frequency fluctuations and stable emission on a single high-gain mode in semiconductor lasers with external optical feedback," Phys. Rev. A., vol. 58, no. 4, pp. 2672-2675, 1998. 